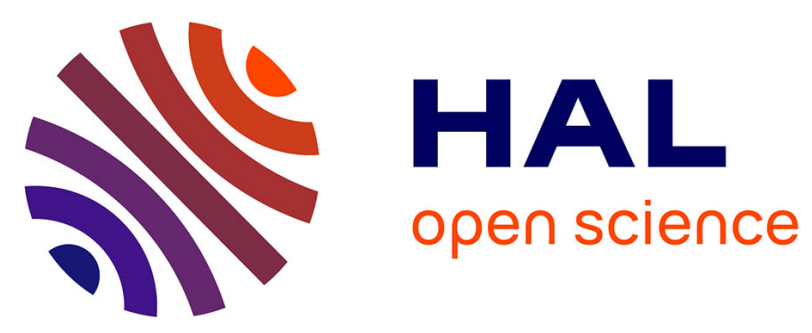

\title{
Prognostic value of global deep white matter DTI metrics for 1-year outcome prediction in ICU traumatic brain injury patients: an MRI-COMA and CENTER-TBI combined study
}

Louis Puybasset, Vincent Perlbarg, Jean Unrug, Didier Cassereau, Damien Galanaud, Grégory Torkomian, Valentine Battisti, Muriel Lefort, Lionel Velly, Vincent Degos, et al.

\section{- To cite this version:}

Louis Puybasset, Vincent Perlbarg, Jean Unrug, Didier Cassereau, Damien Galanaud, et al.. Prognostic value of global deep white matter DTI metrics for 1-year outcome prediction in ICU traumatic brain injury patients: an MRI-COMA and CENTER-TBI combined study. Intensive Care Medicine, In press, 10.1007/s00134-021-06583-z . hal-03479094

\section{HAL Id: hal-03479094 \\ https://hal.sorbonne-universite.fr/hal-03479094}

Submitted on 14 Dec 2021

HAL is a multi-disciplinary open access archive for the deposit and dissemination of scientific research documents, whether they are published or not. The documents may come from teaching and research institutions in France or abroad, or from public or private research centers.
L'archive ouverte pluridisciplinaire HAL, est destinée au dépôt et à la diffusion de documents scientifiques de niveau recherche, publiés ou non, émanant des établissements d'enseignement et de recherche français ou étrangers, des laboratoires publics ou privés. 
Prognostic value of global deep white matter DTI metrics for oneyear outcome prediction in ICU traumatic brain injury patients: an MRI-COMA and CENTER-TBI combined study 


\title{
Authors
}

Louis PUYBASSET, Vincent PERLBARG, Jean UNRUG, Didier CASSEREAU, Damien GALANAUD, Grégory TORKOMIAN, Valentine BATTISTI, Muriel LEFORT, Lionel VELLY, Vincent DEGOS, Guiseppe CITERIO, Éléonore BAYEN, Mélanie PELEGRINI-ISSAC, MRI-COMA investigators ${ }^{1}$, CENTER-TBI MRI participants and MRI only investigators ${ }^{2}$

\author{
1 MRI-COMA investigators \\ Belgium \\ Charlène Aubinet, Manon Carrière, Carol Di Perri, Steven Laureys, Andrea Soddu, Audrey Vanhaudenhuyse: Cyclotron Research Center and Department of \\ Neurology, University of Liège, Liège \\ France \\ Julien Amour: Department of Anesthesiology and Critical Care, Institut de cardiologie, Groupe Hospitalier Pitié-Salpêtrière, Paris \\ Charlotte Arbelot, Jean-Jacques Rouby, Corine Vezinet: Department of Intensive Care, Groupe Hospitalier Pitié-Salpêtrière, Paris \\ Laurent Barral, Jean-Paul Roustan: Department of Intensive Care, University Hospital of Montpellier, Montpellier \\ Nicolas Bruder: Department of Anesthesiology and Critical Care, University Hospital Timone Adultes, Marseille \\ Russel Chabanne, Jean-Michel Constantin: Department of Anesthesiology and Critical Care, University Hospital of Clermont-Ferrand, Clermont-Ferrand \\ Leila Chamard: Department of Neuroradiology, Neurological Hospital, Hospices civils de Lyon, Lyon \\ Jean Chastre, Charles-Edouard Luyt: Department of Medical Intensive Care, Institut de cardiologie, Groupe Hospitalier Pitié-Salpêtrière, Paris \\ Vincent Cottenceau, François Sztark, Olivier Verdonck: Department of Anesthesiology and Critical Care, University Hospital Pellegrin, Bordeaux \\ David Couret: Department of Intensive Care, University Hospital Sud Réunion, Saint-Pierre \\ Frédéric Dailler, Thomas Ritzenthaler: Department of Intensive Care, Neurological Hospital, Hospices civils de Lyon, Lyon \\ Vincent Degos, Louis Puybasset: Department of Neuro Critical Care, Groupe Hospitalier Pitié-Salpêtrière, Paris Francis Bolgert , Nicolas Weiss, Sophie \\ Demeret, Alexandre Demoule, Alexandre Duguet, Thomas Similowski: Department of Respiratory and Critical Care Medicine, Groupe Hospitalier Pitié-Salpê- \\ trière, Paris \\ Pierre-Guy Durand: Department of Intensive Care, Saint-Jean Hospital, Perpignan \\ Damien Galanaud: Department of Neuroradiology, Groupe Hospitalier Pitié-Salpêtrière, Paris \\ Michèle Génestal, Stein Silva: Department of Anesthesiology and Critical Care, University Hospital Purpan, Toulouse \\ Nadine Girard: Department of Neuroradiology, University Hospital Timone Adultes, Marseille \\ Betty Jean: Department of Neuroradiology, University Hospital of Clermont-Ferrand, Clermont-Ferrand \\ Stéphane Kremer: Department of Neuroradiology, University Hospital of Strasbourg, Strasbourg \\ Olivier Langeron, Mathieu Raux: Department of Anesthesiology and Critical Care, Groupe Hospitalier Pitié-Salpêtrière, Paris \\ Nathalie Laquay: Department of Intensive Care, Saint-Anne Hospital, Paris \\ Jean-Albert Lotterie: Neuro-Campus Baudot, Institut des Sciences du Cerveau, Toulouse \\ Nicolas Menjot de Champfleur: Department of Neuroradiology, University Hospital of Montpellier, Montpellier
}


Sandrine Molinier, Thomas Tourdias: Department of Neuroradiology, University Hospital Pellegrin, Bordeaux

Sandrine Mons: Department of Intensive Care, Hospital of Papeete, Papeete

Catherine Oppenheim: Department of Neuroradiology, Saint-Anne Hospital, Paris

Julien Pottecher: Department of Intensive Care, University Hospital of Strasbourg, Strasbourg

Benjamin Rohaut: Neurological Intensive Care Unit, Institut de Neurosciences, Groupe Hospitalier Pitié-Salpêtrière, Paris

Gustavo Sotoares: Department of Neuroradiology, University Hospital Roger Salengro, Lille

Eléonore Tollard: Department of Neuroradiology, University Hospital of Rouen, Rouen

Benoît Veber: Department of Intensive Care, University Hospital of Rouen, Rouen

Emmanuel Vega: Department of Intensive Care, University Hospital Roger Salengro, Lille

Italy

Cristina Agostinis: Department of Neuroradiology, Hospital Papa Giovanni XXIII, Bergamo

Mariagiulia Anglani: Department of Neuroradiology, University Hospital of Padova, Padova

Giuseppe Citerio, Alessia Vargioglu: Department of Anesthesia and Critical Care, Hospital San Gerardo, Monza

Moreno Curti, Francesco Della Corte, Francesca Grossi: Department of Anesthesia and Critical Care, Maggiore della Carita-Emergenza, Novara

Roberto Alberto De Blasi, Livia Errico: Department of Anesthesia and Critical Care, Sant'Andrea Hospital, Roma

Paolo Gritti: Department of Anesthesia and Critical Care, Hospital Papa Giovanni XXIII, Bergamo

Marina Munari: Department of Anesthesia and Critical Care, University Hospital of Padova, Padova

Mirko Patassini: Department of Neuroradiology, Hospital San Gerardo, Monza

\section{CENTER-TBI MRI participants and MRI only investigators}

\section{Belgium}

Véronique De Keyser, Andrew I.R. Maas, Tomas Menovsky, Gregory Van der Steen: Department of Neurosurgery, Antwerp University Hospital and University of Antwerp, Edegem

Bart Depreitere: Department of Neurosurgery, University Hospitals Leuven, Leuven

Alexandre Ghuysen, Emergency Department, CHU, Liège

Steven Laureys, Didier Ledoux, Benoit Misset: Cyclotron Research Center, University of Liège, Liège

Paul M. Parizel: Department of Radiology, University of Antwerp, Edegem

Wim Van Hecke, Thijs Vande Vyvere, Jan Verheyden: icoMetrix NV, Leuven

Finland

Jussi P. Posti, Olli Tenovuo: Division of Clinical Neurosciences, Department of Neurosurgery and Turku Brain Injury Centre, Turku University Hospital and

University of Turku, Turku

Riikka Takala: Perioperative Services, Intensive Care Medicine and Pain Management, Turku University Hospital and University of Turku, Turku

Peter Ylén: VTT Technical Research Centre, Tampere

France

Philippe Azouvi : Raymond Poincaré Hospital, Assistance Publique - Hôpitaux de Paris, Paris

Habib Benali, Vincent Degos, Damien Galanaud, Vincent Perlbarg, Louis Puybasset : Department of Anesthesiology and Critical Care, Pitié -Salpêtrière

Teaching Hospital, Assistance Publique - Hôpitaux de Paris and Sorbonne Université, Paris; Laboratoire d’Imagerie Biomédicale, Sorbonne Université, Inserm, CNRS, Paris

Germany

Ana Kowark, Rolf Rossaint: Department of Anaesthesiology, University Hospital of Aachen, Aachen

Hans Clusmann: Department of Neurosurgery, Medical Faculty RWTH Aachen University, Aachen

Mark Coburn: Department of Anesthesiology and Intensive Care Medicine, University Hospital Bonn, Bonn

Hungary

Krisztina Amrein, József Nyirádi: János Szentágothai Research Centre, University of Pécs, Pécs

Andras Buki, Endre Czeiter: Department of Neurosurgery, Medical School, University of Pécs; Neurotrauma Research Group, János Szentágothai Research

Centre, University of Pécs, Pécs

Erzsébet Ezer, Zoltán Vámos: Department of Anaesthesiology and Intensive Therapy, University of Pécs, Pécs

Noémi Kovács: Hungarian Brain Research Program - Grant No. KTIA_13_NAP-A-II/8, University of Pécs, Pécs 
Abayomi Sorinola, Viktória Tamás: Department of Neurosurgery, University of Pécs, Pécs

Italy

Alessio Caccioppola, Emiliana Calappi, Marco Carbonara, Fabrizio Ortolano, Tommaso Zoerle: Neuro ICU, Fondazione IRCCS Cà Granda Ospedale Maggiore Policlinico, Milano

Giuseppe Citerio: School of Medicine and Surgery, Università Milano Bicocca, Milano, Italy, NeuroIntensive Care, ASST di Monza, Monza

Paolo Persona, Sandra Rossi: Department of Anesthesia \& Intensive Care, Azienda Ospedaliera Università di Padova, Padova

Nino Stocchetti: Department of Pathophysiology and Transplantation, Milan University, and Neuroscience ICU, Fondazione IRCCS Cà Granda Ospedale Maggiore Policlinico, Milano

Norway

Nada Andelic: Division of Surgery and Clinical Neuroscience, Department of Physical Medicine and Rehabilitation, Oslo University Hospital and University of Oslo, Oslo

Lasse Andreassen: Department of Neurosurgery, University Hospital Northern Norway, Troms $\varnothing$

Audny Anke: Department of Physical Medicine and Rehabilitation, University Hospital Northern Norway, Troms $\varnothing$

Live Eikenes: Department of Circulation and Medical Imaging, Norwegian University of Science and Technology, NTNU, Trondheim

Shirin Frisvold: Department of Anesthesiology and Intensive care, University Hospital Northern Norway, Troms $\emptyset$

Asta Haberg, Toril Skandsen: Department of Neuromedicine and Movement Science, Norwegian University of Science and Technology, NTNU, Trondheim;

Department of Physical Medicine and Rehabilitation, St.Olavs Hospital, Trondheim University Hospital, Trondheim

Eirik Helseth: Department of Neurosurgery, Oslo University Hospital, Oslo

Ingeborg Nakken: Department of Radiology and Nuclear Medicine, St. Olavs Hospital, Trondheim University Hospital, Trondheim

Wibeke Nordhøy: Department of Diagnostic Physics, Clinic of Radiology and Nuclear Medicine, Oslo University Hospital, Oslo

Cecilie Roe: Department of Physical Medicine and Rehabilitation, Oslo University Hospital/University of Oslo, Oslo

Olav Roise: Division of Orthopedics, Oslo University Hospital, Oslo, Norway; Institute of Clinical Medicine, Faculty of medicine, University of Oslo, Oslo

Anne Vik: Department of Neuromedicine and Movement Science, Norwegian University of Science and Technology, NTNU, Trondheim; Department of Neurosurgery, St.Olavs Hospital, Trondheim University Hospital, Trondheim

Lars T. Westlye: Norwegian Centre for Mental Disorders Research (NORMENT), Division of Mental Health and Addiction, Oslo University Hospital and Institute of Clinical Medicine, University of Oslo and Department of Psychology, University of Oslo, Oslo

Sweden

Bo-Michael Bellander: Department of Neurosurgery \& Anesthesia \& intensive care medicine, Karolinska University Hospital, Stockholm

Visakh Muraleedharan: Karolinska Institutet, INCF International Neuroinformatics Coordinating Facility, Stockholm

The Netherlands

Kelly Foks: Department of Neurology, Erasmus MC, Rotterdam

Iain Haitsma, Victor Volovici: Department of Neurosurgery, Erasmus MC, Rotterdam

Hester Lingsma: Department of Public Health, Erasmus Medical Center-University Medical Center, Rotterdam

Wilco Peul: Dept. of Neurosurgery, Leiden University Medical Center, Leiden, The Netherlands and Dept. of Neurosurgery, Medical Center Haaglanden, The Hague

Ewout W. Steyerberg: Department of Public Health, Erasmus Medical Center-University Medical Center, Rotterdam; Department. of Department of Biomedical Data Sciences, Leiden University Medical Center, Leiden

\section{United-Kingdom}

Jonathan Coles: Department of Anesthesia \& Neurointensive Care, Cambridge University Hospital NHS Foundation Trust, Cambridge

Marta Correia: Radiology/MRI department, MRC Cognition and Brain Sciences Unit, Cambridge

Ben Glocker, Daniel Rueckert: Department of Computing, Imperial College London, London

Peter J. Hutchinson: Division of Neurosurgery, Department of Clinical Neurosciences, Addenbrooke's Hospital \& University of Cambridge, Cambridge, UK Evgenios Kornaropoulos, David K. Menon, Virginia Newcombe, Sophie Richter, Emmanuel Stamatakis, Guy Williams, Stefan Winzeck: Division of Anaesthesia, University of Cambridge, Addenbrooke's Hospital, Cambridge

USA

Geoffrey Manley: Department of Neurological Surgery, University of California, San Francisco, California

4 


\section{Authors affiliations and coordinates}

Louis PUYBASSET, MD, PhD

- APHP, Sorbonne Université, Hôpital Pitié-Salpêtrière, Neurosurgical Intensive Care Unit, Paris, France

- Sorbonne Université, CNRS, INSERM, Laboratoire d'Imagerie Biomédicale (LIB), Paris, France

- louis.puybasset@aphp.fr

- $\quad(+33) 142162259$

- 0000-0002-6849-1825

Vincent PERLBARG, PhD

- BrainTale SAS, Paris, France

- $\quad$ vincent.perlbarg@braintale.eu

- $\quad$ (+33) 611655478

- $\quad 0000-0002-3438-2479$

Jean UNRUG, MEng

- APHP, Sorbonne Université, Hôpital Pitié-Salpêtrière, Neurosurgical Intensive Care Unit, Paris, France

- $\quad$ Sorbonne Université, CNRS, INSERM, Laboratoire d'Imagerie Biomédicale (LIB), Paris, France

- jeanunrug@gmail.com

Didier CASSEREAU, PhD

- Sorbonne Université, CNRS, INSERM, Laboratoire d'Imagerie Biomédicale (LIB), Paris, France

- didier.cassereau@espci.fr

- $\quad$ (+33) 144279223

- 0000-0003-2412-2144

Damien GALANAUD, MD, PhD

- APHP, Sorbonne Université, Hôpital Pitié-Salpêtrière, Department of Neuroradiology, Paris, France

- $\quad$ Sorbonne Université, CNRS, INSERM, Laboratoire d'Imagerie Biomédicale (LIB), Paris, France

- damien.galanaud@aphp.fr

- $\quad$ (+33) 142163501

- $0000-0002-9285-8121$

Grégory TORKOMIAN, MEng

- APHP, Sorbonne Université, Hôpital Pitié-Salpêtrière, Neurosurgical Intensive Care Unit, Paris, France

- gregory.torkomian@aphp.fr

- $\quad(+33) 142163423$

- $0000-0003-0175-945 \mathrm{X}$

Valentine BATTISTI, PhD

- APHP, Sorbonne Université, Hôpital Pitié-Salpêtrière, Neurosurgical Intensive Care Unit, Paris, France

- $\quad$ valentine.battisti@aphp.fr

- $\quad$ (+33) 142163701

- 0000-0003-3041-7141

Muriel LEFORT, MSc

- Sorbonne Université, CNRS, INSERM, Laboratoire d'Imagerie Biomédicale (LIB), Paris, France

- muriel.lefort@inserm.fr 
- $\quad(+33) 144279308$

- $0000-0001-7820-3754$

Lionel VELLY, MD, PhD

- AP-HM, Aix Marseille University, Department of Anesthesiology and Critical Care Medicine, University Hospital Timone, Marseille, France

- Aix Marseille University, CNRS, Institute of Neuroscience Timone, UMR7289, Marseille, France

- LionelJean.VELLY@ap-hm.fr

- $\quad$ (+33) 413429471

- $\quad 0000-0001-5009-1711$

Vincent DEGOS, MD, PhD

- APHP, Sorbonne Université, Hôpital Pitié-Salpêtrière, Department of Anesthesia, Critical Care and Peri-Operative Medicine, Paris, France.

- Sorbonne Université, Clinical Research Group 29, Paris, France.

- INSERM UMR 1141, Paris, France

- $\quad$ vincent.degos@inserm.fr

- $\quad$ (+33) 184827179

- $0000-0002-6540-5725$

Giuseppe CITERIO, MD

- University of Milano-Bicocca, Department of Medicine and Surgery, Monza, Italy

- $\quad$ ASST- Monza, San Gerardo Hospital, Neurointensive care unit, Department of Emergency and Urgency, Monza, Italy

- giuseppe.citerio@unimib.it

- $\quad(+39) 3490956697$

- 0000-0002-5374-3161

Éléonore BAYEN, MD, PhD

- $\quad$ APHP, Sorbonne Université, Hôpital Pitié-Salpêtrière, Rehabilitation Unit, Paris, France

- Sorbonne Université, CNRS, INSERM, Laboratoire d'Imagerie Biomédicale (LIB), Paris, France

- eleonore.bayen@aphp.fr

- $\quad(+33) 142161101$

- 0000-0002-0286-5521

Mélanie PELEGRINI-ISSAC, PhD

- $\quad$ Sorbonne Université, CNRS, INSERM, Laboratoire d'Imagerie Biomédicale (LIB), Paris, France

- melanie.pelegrini@inserm.fr

- $\quad$ (+33) 144279309

- $0000-0003-3260-5795$ 


\section{Corresponding author}

Louis Puybasset, M.D., Ph.D.

Department of Anesthesiology and Intensive Care

Groupe Hospitalier Pitié-Salpêtrière

Assistance Publique-Hôpitaux de Paris

47-83 Boulevard de l'hôpital

75013 Paris

France

Tel: +33 142162259

Email: louis.puybasset@aphp.fr 


\section{Take home message}

We developed a prognostic score based on MR diffusion metrics measured in deep white matter between 7 and 35 days after onset, to assess one-year outcome in ICU patients with severe traumatic brain injury. The score identified one in two patients who eventually had an unfavorable outcome, and two-thirds of the patients who actually had a favorable outcome at one year after the injury. For both conditions, specificity was above $95 \%$, a value compatible with a personalized decision-making process in ICU. 


\begin{abstract}
Purpose: A reliable tool for outcome prognostication in severe traumatic brain injury (TBI) would improve intensive care unit (ICU) decision-making process by providing objective information to caregivers and family. This study aimed at designing a new classification score based on magnetic resonance (MR) diffusion metrics measured in the deep white matter between day 7 and day 35 after TBI to predict 1-year clinical outcome.
\end{abstract}

Methods: Two multicenter cohorts (29 centers) were used. MRI-COMA cohort (NCT00577954) was split into MRI-COMA-Train (50 patients enrolled between 2006 and mid-2014) and MRI-COMA-Test (140 patients followed up in clinical routine from 2014) sub-cohorts. These latter patients were pooled with 56 ICU patients (enrolled from 2014 to 2020) from CENTER-TBI cohort (NCT02210221). Patients were dichotomised depending on their one-year Glasgow outcome scale extended (GOSE) score: GOSE 1-3, unfavorable outcome (UFO); GOSE 4-8, favorable outcome (FO). A support vector classifier incorporating fractional anisotropy and mean diffusivity measured in deep white matter, and age at the time of injury was developed to predict whether the patients would be either UFO or FO.

Results: The model achieved an area under the ROC curve of 0.93 on MRI-COMA-Train training dataset, and $49.0 \%$ sensitivity for $96.8 \%$ specificity in predicting UFO and $58.5 \%$ sensitivity for $97.1 \%$ specificity in predicting FO on the pooled MRI-COMA-Test and CENTER-TBI validation datasets.

Conclusion: The model successfully identified, with a specificity compatible with a personalized decision-making process in ICU, one in two patients who had an unfavorable outcome at one year after the injury, and two-thirds of the patients who experienced a favorable outcome.

\title{
Keywords
}

traumatic brain injury

outcome

prognosis

diffusion tensor imaging

deep white matter 


\section{Declarations}

\section{Funding}

The MRI-COMA trial was funded by independent research grants from non-profit or governmental agencies: French Ministry of Health, Paris, France (Programme Hospitalier de Recherche Clinique 2005 \#051061), and the French National Agency for Research (ANR) for the program "Investissements d'avenir" ANR-10-IAIHU-06 (to the Brain and Spine Institute); Italian Ministry of health and Regione Lombardia (Ricerca Finalizzata 2010 - RF-2010-2319503).

CENTER-TBI data used in preparation of this manuscript were obtained in the context of CENTERTBI, a large collaborative project with the support of the European Union 7th Framework program (EC grant 602150). Additional funding was obtained from the Hannelore Kohl Stiftung (Germany), from OneMind (USA) and from Integra LifeSciences Corporation (USA).

\section{Conflicts of interest/Competing interests}

DG, LP, and VP share together with institutions (AP-HP, INSERM, CNRS, and Sorbonne University) the property of patent WO2012160316, which partly covers the research of the present study. DC, DG, LP and VP are co-founders and members of the advisory board of Braintale SAS in terms approved by Sorbonne Université (Paris, France) in accordance with its policy on objectivity in research. GC is Editor in Chief of Intensive Care Medicine. The other authors do not have any conflict of interest to disclose.

\section{Availability of data and material}

Not applicable

\section{Code availability}

Not applicable

\section{Authors' contributions following CRediT taxonomy}

- Conceptualization: LP, VP, LV, EB, MPI

- Methodology: MPI, VP, JU

- Software: MPI, VP, JU

- Validation: all

- Formal analysis: MPI, VP, JU

- Investigation: VB, GC, VD, DG, LP, GT

- Resources: GC, VD, DG, LP

- Data curation: VB, DC, ML, GT, JU

- Writing - Original draft: MPI, VP, EB, LP

- Writing - Review \& editing: all

- Visualization: MPI, JU 
- Supervision: LP, VD, VP, MPI

- Project administration: LP, GT, MPI

- Funding acquisition: LP

All approved the final version.

\section{Ethics approval}

Both MRI-COMA (NCT00577954) and CENTER-TBI (NCT02210221) studies were conducted in accordance with all relevant laws of the EU if directly applicable or of direct effect and all relevant laws of the country where the Recruiting sites were located, including but not limited to, the relevant privacy and data protection laws and regulations (the "Privacy Law"), the relevant laws and regulations on the use of human materials, and all relevant guidance relating to clinical studies from time to time in force including, but not limited to, the ICH Harmonised Tripartite Guideline for Good Clinical Practice (CPMP/ICH/135/95) ("ICH GCP”) and the World Medical Association Declaration of Helsinki entitled "Ethical Principles for Medical Research Involving Human Subjects". Ethical approval was obtained for each recruiting site.

For details regarding MRI-COMA, the Direction de la Recherche Clinique et de l'Innovation (DRCI), Assistance Publique Hôpitaux de Paris (Paris, France) can be contacted: secretariat-direction.drc@aphp.fr

For CENTER-TBI, the list of sites, Ethical Committees, approval numbers and approval dates can be found on the website: https://www.center-tbi.eu/project/ethical-approval

\section{Consent to participate}

- CENTER-TBI: Informed Consent by the patients and/or the legal representative/next of kin was obtained, accordingly to the local legislations, for all patients recruited in the Core Dataset of CENTER-TBI and documented in the e-CRF.

- MRI-COMA: Informed Consent by the patients and/or the legal representative/next of kin was obtained, accordingly to the local legislations.

\section{Consent for publication}

Not applicable

\section{Acknowledgments}

We would like to thank patients, relatives, and clinical and research staff at all trial sites. 


\section{Introduction}

Coma has multiple etiologies among which traumatic brain injury (TBI) constitutes a major cause of death and disability for about 2.5 million people per year in Europe [1]. Some patients make a complete recovery from coma, but others retain persistent neurologic deficit with possible permanent disorder of consciousness (DOC), namely minimally conscious or vegetative state [2]. There is a worldwide urgent need for accurate prognostication of permanent DOC following TBI. Regardless of cultural or reimbursement issues, improved prognostic in the intensive care unit (ICU) would allow better titration of care, resource allocation and family support.

Prognostication of comatose patients with TBI in ICU remains challenging, particularly for those who do not regain consciousness by day 7 post-injury. Most of the existing prognosis tools such as the international mission for prognosis and analysis of clinical trials (IMPACT) prognosis calculator [3] or the corticosteroid randomisation after significant head injury (CRASH) model [4] include clinical (e.g. initial Glasgow coma scale [GCS], age, blood pressure) and computerized tomography (CT) predictors. These widely validated models $[3,5]$ concern patients in ultra-early stages (within the first 72 hours following admission, within the first week at the latest) and predict 14-day mortality and/or 6-month outcome, often measured as the probability of survival, the Glasgow outcome scale (GOS) or the GOS extended (GOSE). Indeed, a significant proportion of comatose patients either die or awake during this early phase, thereby clarifying the immediate decision-making process.

However, reporting of late recovery of consciousness in recent studies has highlighted the risk of inappropriate early prognostication $[6,7]$. In clinical practice, predicting patients' outcome well after 6 months and at least one year post injury is a key issue for patients who remain unresponsive after day 7 . In the absence of a validated DOC prognostication tool, ethical decisions related to withdrawal of lifesustaining treatments (WLST) depend on the service/hospital/country where the patient is treated. Reliable prognostication would limit between-center/between-country disparities in the decision-making process by providing objective shared information to professional caregivers and family.

During the past decade, multimodal magnetic resonance imaging (MRI) - especially diffusion-tensor imaging (DTI) [8] - has emerged as a promising prognostication modality [9]. Studies have found 12 
consistent changes in regional diffusion measures (fractional anisotropy [FA] and mean diffusivity [MD]) that have high predictive values of long-term recovery of consciousness after TBI and cardiac arrest [10-12]. FA is usually related to white matter fiber integrity, while MD rather pertains to the amount of water in the extracellular space [13]. The objective of our study was to assess whether global measures of FA and MD within the deep white matter of the brain would capture severe brain alterations within the first five weeks following TBI and successfully identify patients with an eventual favorable 1-year outcome (FO, GOSE 4-8) $[14,15])$ and patients with an unfavorable outcome (UFO, GOSE 13). Age at the time of the injury was incorporated in the prognostic model since older age is associated with a worsening outcome after severe TBI [16] and diffusion metrics (in particular MD) vary with normal ageing [17].

As obtaining DTI data from intubated patients is not accessible to all ICU, existing studies have involved rather small cohorts of about 100 patients at most $[10,18]$ and predicting models have lacked validation on multicenter cohorts external to the training dataset. Here, the proposed model was validated on an external dataset comprising a subset of the European cohort MRI-COMA and a subset of the observational cohort CENTER-TBI [1]. 


\section{Methods}

TRIPOD reporting guidelines were followed [19].

\section{Study design}

Two different prospective, multicenter cohorts of TBI patients only were used in this study. At least five healthy volunteers were recruited in each center for calibration purposes (Online Resource 4). The studies were approved by local ethics committees in each participating country.

\section{$\underline{\text { MRI-COMA cohort }}$}

MRI-COMA (ClinicalTrials.gov identifier: NCT00577954) is a cohort study assessing outcome with multimodal MRI in comatose patients of various etiologies. Patients were enrolled in 17 ICUs in France, Belgium, and Italy. The cohort was split into two sub-cohorts: MRI-COMA-Train included patients enrolled between October 2006 and June 2014; MRI-COMA-Test included patients followed up in clinical routine between July 2014 and September 2020. Interim analyses have already been published for patients with cardiac arrest [20,21].

\section{CENTER-TBI cohort}

CENTER-TBI (ClinicalTrials.gov identifier: NCT02210221) is a cohort study aimed at better characterizing TBI as a disease in a European context, and at identifying effective clinical interventions for managing TBI [1]. Patients were enrolled in 60 ICUs in 22 countries in Europe and Israel between December 2014 and March 2020. Only patients admitted directly to the ICU ("ICU stratum") who undertook MR scans between day 7 and day 35 after the injury ("2-3 weeks stratum") were considered.

\section{Inclusion and exclusion criteria}

Patients eligible for inclusion were aged between 18 and 70 years at the time of the brain injury. In the MRI-COMA cohort specifically, only those patients unable to respond to simple orders unexplained by sedation for at least 7 days after the event were included. The main exclusion criteria were contraindication to MRI and pre-existing neurological disorders that would confound outcome assessments. 
Inclusion and exclusion criteria for MRI-COMA and CENTER-TBI cohorts are listed in Online Resource 1 .

\section{Data acquisition}

Demographic and clinical information including initial severity data (GCS) were collected prospectively. MRI acquisitions were performed between day 7 and day 35 after the event on $1.5 \mathrm{~T}$ or $3 \mathrm{~T}$ scanners from three manufacturers: GE Medical Systems (Milwaukee, WI), Siemens Medical Solutions (Erlangen, Germany), and Philips Medical Systems (Eindhoven, The Netherlands). In addition to a conventional 3D T1-weighted sequence, a diffusion-weighted imaging (DWI) sequence with a minimum of 29 gradient directions was acquired. Sequence parameters are given in Online Resource 3 . The healthy volunteers underwent the same imaging protocol as the patients. For MRI-COMA trial, anonymized MR source images were transferred to the coordinating center. CENTER-TBI data were collected through the Quesgen e-CRF (Quesgen Systems Inc, USA) hosted on the INCF platform and extracted via the INCF Neurobot tool (INCF, Sweden). Version Center Core 2.1 of CENTER-TBI dataset was used in this study. Image data collection was facilitated and hosted on the Icometrix platform (Icometrix, Leuven).

\section{DWI data processing}

All DWI data analysis steps are described in Online Resource 4. They include quality checking, computing and averaging FA and MD maps in deep white matter, and calibrating average values for each patient using measures from healthy volunteers to reduce between-center variability [22], leading to $\overline{\mathrm{FA}}_{\text {deep }}$ and $\overline{\mathrm{MD}}_{\text {deep }}$ values, respectively.

\section{Patient outcome}

The GOSE score was assessed at ICU discharge and between 6 months and one year post-injury. This latter value was considered as the outcome for the prediction model (denoted as "1-year GOSE"), as follows: 1) 1-year unfavorable outcome (UFO, GOSE from 1 to 3), corresponding to death, vegetative 
state, or lower severe disability; and 2) 1-year favorable outcome (FO, GOSE from 4 to 8), corresponding to upper severe disability to good recovery.

Repeated GOSE assessment during clinical routine follow-up (1 to 5 years post-injury) was available for a majority of patients in the MRI-COMA cohort. GOSE score was also collected at 2 years postinjury for CENTER-TBI patients who underwent repeated neuropsychological testing.

\section{Prognostic score derivation}

The predictive model was implemented using a supervised learning approach involving a support vector classifier (see Online Resource 5 for details). First, patients of the MRI-COMA-Train sub-cohort, used as the training dataset, were classified in two outcome categories UFO or FO: each patient was characterized by a prediction score CSCORE given his/her $\overline{\mathrm{FA}}_{\text {deep }}, \overline{\mathrm{MD}}_{\text {deep }}$ and age values. We then defined optimal thresholds for CSCORE in order to enforce a strong specificity for FO (95\%) and UFO (99\%) prediction, while maximizing sensitivity for each outcome prediction. The choice of deriving decision rules with such a low false-positive rate in predicting UFO at the expense of sensitivity was driven by the need for highest confidence in the difficult decision-making process. This yielded two optimal CSCORE cut-off values CSCORE $E_{\text {low }}$ and $\mathrm{CSCORE}_{\text {high. }}$ Patients with CSCORE lower than $\mathrm{CSCORE}_{\text {low }}$ were predicted to have a UFO with more than $99 \%$ specificity; patients with CSCORE higher than CSCORE $_{\text {high }}$ were predicted to have a FO with more than 95\% specificity; patients with CSCORE between $\mathrm{CSCORE}_{\text {low }}$ and $\mathrm{CSCORE}_{\text {high }}$ were predicted neither UFO nor FO and remained in a so-called "gray zone".

The prediction model was subsequently validated by pooling the two cohorts MRI-COMA-Test and CENTER-TBI to a single external validation dataset. Validation was also assessed after excluding patients who underwent WLST.

\section{Statistical analyses}

Means and standard deviations were used for continuous variables (age, delay from primary injury to MRI exam, length of stay in ICU, $\overline{\mathrm{FA}}_{\text {deep }}$ and $\overline{\mathrm{MD}}_{\text {deep }}$ ); medians and interquartile ranges were used for 
GCS and GOSE. Group differences between training and validation datasets were assessed with unpaired two-sample T-tests, Fisher's exact test, Wilcoxon-Mann-Whitney U tests, and MANOVA (Pillai's trace statistic) where appropriate ( $<<0.05$ considered as significant). All analyses were performed using $\mathrm{R}$ version 4.0.2 [23]. 


\section{Results}

\section{Patient cohorts}

A total of 303 patients fulfilled the inclusion criteria. Among them, 60 had decompressive craniectomy (DC). Quality check failed in 18/60 (30.0\%) patients with DC (due to misregistration of FA and MD maps), but only in 39/243 (16.0\%) patients without DC. Following data quality check, 246 patients were finally included in the study: 190 from the MRI-COMA cohort (50 in the training dataset and 140 in the validation dataset, from 17 centers) and 56 patients from the CENTER-TBI cohort (in the validation dataset, from 12 centers) (Figure 1).

- Figure 1 about here -

Baseline characteristics for the training and validation datasets are given in Tables 1 and 2. No significant difference was found between both datasets in terms of age ( $\mathrm{T}$-test $\mathrm{T}=-1.28, \mathrm{p}=0.20$ ), gender (Fisher's exact test odds ratio $=1.07, \mathrm{p}=0.85$ ), mean delay to MRI ( $\mathrm{T}$-test $\mathrm{T}=1.76, \mathrm{p}=0.08$ ), GCS (Wilcoxon $\mathrm{W}=4422, \mathrm{p}=0.28$ ), and GOSE score (Wilcoxon $\mathrm{W}=4361, \mathrm{p}=0.22$ ). Length of stay (LOS) in the ICU was significantly lower in the validation dataset than in the training dataset due to shorter LOS of CENTER-TBI patients (Table 2, T-test $\mathrm{T}=4.25, \mathrm{p}<0.001$ ). $\overline{\mathrm{FA}}_{\text {deep }}$ and $\overline{\mathrm{MD}}_{\text {deep }}$ values were significantly correlated (Spearman $\mathrm{r}=-0.46, \mathrm{p}<0.001)$ but did not differ between datasets (MANOVA, Pillai's trace $=0.008, \mathrm{~F}=0.97, \mathrm{p}=0.38)$.

- Table 1 about here -

- Table 2 about here -

A total of 207 healthy controls were included. Age did not differ significantly between controls and patients in the training dataset ( $\mathrm{T}$-test $\mathrm{T}=-0.70, \mathrm{p}=0.49$ ) but differed in the validation dataset ( $\mathrm{T}$-test $\mathrm{T}=-2.38, \mathrm{p}=0.02$ ) due to the lower age of controls compared with patients. Mean age and gender for patients and controls in each center are detailed in Online Resource 2. 


\section{Classification performance}

Regarding the training dataset, the prediction model achieved an area under the receiver operating characteristic (ROC) curve of 0.93 , yielding $46.7 \%$ sensitivity for $100 \%$ specificity in predicting UFO (CSCORE $E_{\text {low }}$ cut-off value: 0.19 ) and $55.0 \%$ sensitivity for $96.7 \%$ specificity in predicting $\mathrm{FO}$ $\left(\mathrm{CSCORE}_{\text {high }}\right.$ cut-off value: 0.65$)$. On the validation dataset, the classifier achieved an area under the ROC curve of 0.89 , with $49.0 \%$ sensitivity for $96.8 \%$ specificity in predicting UFO ( $71.9 \%$ accuracy) and $58.5 \%$ sensitivity for $97.1 \%$ specificity in predicting FO (78.6\% accuracy). A complete list of performance metrics with $95 \%$ confidence intervals is provided in Online Resource 6. Figure 2 illustrates 1-year GOSE category compared with that predicted by the model. The lower CSCORE, the higher the proportion of actual UFO patients. Patients in the "red area" in Figure 2 (CSCORE less than CSCORE low, $_{\text {, }}$ $\mathrm{N}=53$ ) were predicted to have a UFO, patients in the "green area" (CSCORE higher than $\mathrm{CSCORE}_{\text {high }}$, $\mathrm{N}=58)$ were predicted to have a FO, and patients in the "gray zone" (N=85) did not meet either specificity criterion and were classified as neither UFO nor FO patients. Patients with red dots in Figure 2 ( $\mathrm{N}=102)$ were actually assessed with UFO. Patients with green '+' ( $\mathrm{N}=94)$ were assessed with FO; 3 patients (3.2\%) were predicted to be UFO by the model while their GOSE was actually 4 one year after the injury, but no further follow-up data was available for these patients after one year (see Figure 9c in Online Resource 9).

- Figure 2 about here-

The delay between the injury and the MRI session did not influence the performance of the model (Online Resource 7). Once validated after excluding the 50 patients who underwent WLST, the prediction model achieved $32.7 \%$ sensitivity for $96.8 \%$ specificity in predicting UFO and $58.5 \%$ sensitivity for $96.1 \%$ specificity in predicting FO.

\section{Evolution of GOSE score after one year}

- Figure 3 about here- 
Figure 3 shows the evolution of GOSE score for the patients for whom follow-up GOSE assessments were available after 1 year (data for all patients are available in Online Resource 9).

Seventy patients were predicted to have a FO. Four were assessed with UFO, two of them died (Figure 3a) and two others were lost to follow-up. Sixty-six (94.3\%) patients were assessed with FO, 33 were followed up (Figure 3a) and remained with FO.

One hundred and nine patients were classified in the "gray zone", i.e. predicted with neither UFO nor FO. Sixty-four (58.7\%) were assessed UFO; 40 patients had follow-up available (Figure 3b): 10 remained with UFO, 4 patients improved from GOSE 3 to 4, 26 died. Forty-five (41.3\%) patients were assessed FO; 19 patients were followed up (Figure 3b) and remained with FO.

Finally, sixty-seven patients were predicted to have a UFO. Three were assessed with FO (GOSE 4 at one year) but none of them had long-term follow-up data available (Online Resource 9, Figure 9c). Sixty-four (95.5\%) were assessed with UFO; 56 patients had follow-up data available (Figure 3c): 3 patients remained with UFO, 51 patients died within the first year and 2 patients died later. 


\section{Discussion}

The present study focused on severe TBI patients who did not recover consciousness by day 7. CSCORE is indeed aimed at addressing the prognosis of those patients who neither emerge from coma, neither die after day 7. Predicting long-term recovery in these patients raises major ethical and care issues. Early withdrawal of care eliminates the possibility of eventual recovery of consciousness while prolonged intervention increases the risk of permanent DOC or severe disability. Most prognosis tools designed to help clinical decision are devoted to predicting UFO. The score proposed in this paper was optimized to predict not only UFO with $99 \%$ specificity, but also FO with $95 \%$ specificity, thus facilitating informed clinical decision and counselling of families by providing quantitative information. CSCORE relies on the combination of patient age with two observer-independent metrics from diffusion-weighted MRI $\left(\overline{\mathrm{FA}}_{\text {deep }}\right.$ and $\left.\overline{\mathrm{MD}}_{\text {deep }}\right)$, which quantify global deep white matter microstructural changes that are not visible on conventional structural MRI data (normal appearing white matter). A decrease in FA can be induced by different pathophysiological processes [24], including myelin damage occurring after TBI and axonal degeneration observed after ischemic anoxic brain injury. On the other hand, seemingly contradictory variations of MD in TBI patients have been reported, with increases suggesting vasogenic edema while decreases suggest cytotoxic edema [25]. Besides, younger age and higher cognitive reserve have been associated with better cognitive recovery in adults with complicated mild-to-severe TBI [26].

CSCORE was externally validated on a dataset independent of the training dataset but sharing similar characteristics in terms of age, mean delay to MRI, and mean metrics values. The overall predictive performance obtained by using external validation for 1-year UFO prediction (specificity/sensitivity: 96.8\%/49.0\%) and FO prediction (specificity/sensitivity: 97.1\%/58.5\%) is compatible with reliable and personalized clinical use. Since we enforced maximal specificity in predicting either FO or UFO, the proposed score differs from the few other methods predicting survival or GOSE at one year based either on clinical and CT data ([27] report an area under the ROC curve of 0.83) or on diffusion MRI. In the patients fulfilling the inclusion criteria of the present study, CSCORE also outperformed the conventional "IMPACT core+CT+lab" score, which yielded an area under the ROC curve of 0.64 only (Online 
Resource 8). Recently, a prediction score relying on quantitative EEG has reported a sensitivity of $100 \%$ in predicting poor outcome, but with a specificity of $75 \%$ only [28].

Inclusion criteria differed slightly between the two sub-cohorts of the validation dataset, with patients in CENTER-TBI sub-cohort being less severe than those in MRI-COMA sub-cohort in terms of initial GCS (Kruskal-Wallis test followed by post-hoc Bonferroni-corrected Dunn's test, $\mathrm{p}<0.001$ ). However, including those patients with a better prognosis can be seen as a strength of our approach in that it demonstrates how CSCORE behaves in everyday life for any type of patient.

CSCORE achieved a global sensitivity close to $50 \%$. Consequently, no informative value could be obtained for one out of every two patients since $43 \%$ of the patients in the validation dataset were classified in the "gray zone". In our view, this is not a weakness of the algorithm. On the contrary, this means that the model classifies $57 \%$ of the patients with a specificity higher than $95 \%$, which is suitable for a future personalized medicine tool while the patient is still in the ICU and, in particular, as a support for WLST decision-making process. To our knowledge, in this population, no tool exists that is able to provide such performance. Regarding the patients in the "gray zone", as of today, our recommendation is to monitor their clinical evolution and, in absence of recovery, perform a second MRI 15 days apart.

The delay range between the injury and the MRI session chosen in this study may be considered as unusually large. Indeed, one could argue that a large delay might influence diffusion metrics for pathophysiological reasons, although we do not have at this stage serial data to assess that. However, such a large delay range is compatible with the average length of stay in ICU observed in severe TBI patients [29].

Follow-up results over several years further enabled to demonstrate the robustness of such a prognostic score. Indeed, nearly all the patients correctly predicted to be either UFO or FO maintained in the same outcome category over time, showing the benefit of CSCORE in informing clinical decision. Further investigation is needed to improve prognostication for patients in the so-called "gray zone". Alternative approaches might implement artificial intelligence techniques, involving analyzing specific regions of the brain and obtaining metrics from more advanced methods than DTI such as diffusional kurtosis 
imaging or neurite orientation dispersion and density imaging (NODDI) [30, 31]. Additional biomarkers such as genetic markers, quantitative EEG, repeated MRI and biology in case of persistent absence of response to simple orders might improve prediction [32]. Further validation studies in a multimodal context remain necessary.

Early withdrawal of care remains the major determinant of in-hospital mortality and may be the basis for a self-fulfilling prophecy of poor outcome [33]. In our study, investigators were not blinded to the morphological MRI and WLST practices were not standardized among centers, which could induce a bias in selecting patients for WLST. We therefore also reported classification results from the validation data after excluding patients who underwent WLST. As one might expect, sensitivity in predicting UFO decreased from $49.0 \%$ to $32.7 \%$ for a similar specificity of $96.8 \%$ while specificity in predicting FO decreased from $97.1 \%$ to $96.1 \%$ for a similar sensitivity of $58.5 \%$. We however acknowledge that excluding WLST patients does not totally solve out this issue: the only real way of avoiding this bias would be to forbid WLST, which is impossible and unethical in clinical practice.

Several technical limitations may also slow down the spreading of this approach in clinical context. First, MRI scanner calibration currently requires data from a handful of controls undergoing exactly the same protocol as the patients, which is further complicated by the regular updates of MRI sequences and the difficulties associated with scanning healthy subjects using clinical equipment. Developing an MRI phantom suitable for calibrating DTI metrics would be a solution to bypass these difficulties but such devices are not routinely available yet [34]. Secondly, transportation and monitoring of ventilated patients to the MRI is complex and may requires immobilization by means of sedation with neuromuscular blocker. Finally, effort must be put into standardizing protocols in MRI centers and reinforcing adherence to good practice procedures to optimize the management of TBI patients. Further implementation of common data elements including DWI acquisition is necessary to harmonize data collection across TBI clinical studies [35].

\section{Conclusion}

We developed a prognostic score based on MR diffusion metrics measured in deep white matter between 7 and 35 days after onset, to assess one-year outcome in ICU patients with severe traumatic brain injury. 23 
The score identified one in two patients who eventually had an unfavorable outcome, and two-thirds of the patients who actually had a favorable outcome at one year after the injury. For both conditions, specificity was above $95 \%$, a value compatible with personalized decision-making process in ICU. We are confident in the added value of such a tool within the framework of a multimodal evaluation to facilitate management of prolonged comatose patients in ICU. 


\section{References}

1. Maas AIR, Menon DK, Steyerberg EW, et al (2015) Collaborative European NeuroTrauma Effectiveness Research in Traumatic Brain Injury (CENTER-TBI): a prospective longitudinal observational study. Neurosurgery 76:67-80. https://doi.org/10.1227/NEU.0000000000000575

2. Giacino JT, Fins JJ, Laureys S, Schiff ND (2014) Disorders of consciousness after acquired brain injury: the state of the science. Nat Rev Neurol 10:99-114. https://doi.org/10.1038/nrneurol.2013.279

3. Steyerberg EW, Mushkudiani N, Perel P, et al (2008) Predicting outcome after traumatic brain injury: development and international validation of prognostic scores based on admission characteristics. PLoS Med 5:e165; discussion e165. https://doi.org/10.1371/journal.pmed.0050165

4. MRC CRASH Trial Collaborators (2008) Predicting outcome after traumatic brain injury: practical prognostic models based on large cohort of international patients. BMJ 336:425-429. https://doi.org/10.1136/bmj.39461.643438.25

5. Gravesteijn BY, Nieboer D, Ercole A, et al (2020) Machine learning algorithms performed no better than regression models for prognostication in traumatic brain injury. J Clin Epidemiol 122:95-107. https://doi.org/10.1016/j.jclinepi.2020.03.005

6. Estraneo A, Moretta P, Loreto V, et al (2010) Late recovery after traumatic, anoxic, or hemorrhagic long-lasting vegetative state. Neurology 75:239-245. https://doi.org/10.1212/WNL.0b013e3181e8e8cc

7. Baricich A, de Sire A, Antoniono E, et al (2017) Recovery from vegetative state of patients with a severe brain injury: a 4-year real-practice prospective cohort study. Funct Neurol 32:131-136. https://doi.org/10.11138/fneur/2017.32.3.131

8. Basser PJ, Mattiello J, LeBihan D (1994) MR diffusion tensor spectroscopy and imaging. Biophys J 66:259-267. https://doi.org/10.1016/S0006-3495(94)80775-1 
9. Stevens RD, Hannawi Y, Puybasset L (2014) MRI for coma emergence and recovery. Curr Opin Crit Care 20:168-173. https://doi.org/10.1097/MCC.0000000000000069

10. Sidaros A, Engberg AW, Sidaros K, et al (2008) Diffusion tensor imaging during recovery from severe traumatic brain injury and relation to clinical outcome: a longitudinal study. Brain J Neurol 131:559-572. https://doi.org/10.1093/brain/awm294

11. Perlbarg V, Puybasset L, Tollard E, et al (2009) Relation between brain lesion location and clinical outcome in patients with severe traumatic brain injury: a diffusion tensor imaging study using voxel-based approaches. Hum Brain Mapp 30:3924-3933. https://doi.org/10.1002/hbm.20817

12. Newcombe V, Chatfield D, Outtrim J, et al (2011) Mapping traumatic axonal injury using diffusion tensor imaging: correlations with functional outcome. PloS One 6:e19214. https://doi.org/10.1371/journal.pone.0019214

13. O'Donnell LJ, Westin C-F (2011) An introduction to diffusion tensor image analysis. Neurosurg Clin N Am 22:185-viii. https://doi.org/10.1016/j.nec.2010.12.004

14. Jennett B, Bond M (1975) Assessment of outcome after severe brain damage. Lancet Lond Engl 1:480-484. https://doi.org/10.1016/s0140-6736(75)92830-5

15. Wilson JT, Pettigrew LE, Teasdale GM (1998) Structured interviews for the Glasgow Outcome Scale and the extended Glasgow Outcome Scale: guidelines for their use. J Neurotrauma 15:573-585. https://doi.org/10.1089/neu.1998.15.573

16. Hukkelhoven CWPM, Steyerberg EW, Rampen AJJ, et al (2003) Patient age and outcome following severe traumatic brain injury: an analysis of 5600 patients. J Neurosurg 99:666-673. https://doi.org/10.3171/jns.2003.99.4.0666

17. Cox SR, Ritchie SJ, Tucker-Drob EM, et al (2016) Ageing and brain white matter structure in 3,513 UK Biobank participants. Nat Commun 7:13629. https://doi.org/10.1038/ncomms13629 
18. Galanaud D, Perlbarg V, Gupta R, et al (2012) Assessment of white matter injury and outcome in severe brain trauma: a prospective multicenter cohort. Anesthesiology 117:1300-1310. https://doi.org/10.1097/ALN.0b013e3182755558

19. Collins GS, Reitsma JB, Altman DG, Moons KGM (2015) Transparent reporting of a multivariable prediction model for individual prognosis or diagnosis (TRIPOD): the TRIPOD statement. BMJ 350:g7594. https://doi.org/10.1186/s12916-014-0241-z

20. Luyt C-E, Galanaud D, Perlbarg V, et al (2012) Diffusion tensor imaging to predict long-term outcome after cardiac arrest: a bicentric pilot study. Anesthesiology 117:1311-1321. https://doi.org/10.1097/ALN.0b013e318275148c

21. Velly L, Perlbarg V, Boulier T, et al (2018) Use of brain diffusion tensor imaging for the prediction of long-term neurological outcomes in patients after cardiac arrest: a multicentre, international, prospective, observational, cohort study. Lancet Neurol 17:317-326. https://doi.org/10.1016/S1474-4422(18)30027-9

22. Pagani E, Hirsch JG, Pouwels PJW, et al (2010) Intercenter differences in diffusion tensor MRI acquisition. J Magn Reson Imaging JMRI 31:1458-1468. https://doi.org/10.1002/jmri.22186

23. R Core Team (2019) R: A Language and Environment for Statistical Computing. R Foundation for Statistical Computing, Vienna, Austria

24. Budde MD, Kim JH, Liang H-F, et al (2007) Toward accurate diagnosis of white matter pathology using diffusion tensor imaging. Magn Reson Med 57:688-695. https://doi.org/10.1002/mrm.21200

25. Hutchinson EB, Schwerin SC, Avram AV, et al (2018) Diffusion MRI and the detection of alterations following traumatic brain injury. J Neurosci Res 96:612-625. https://doi.org/10.1002/jnr.24065 
26. Fraser EE, Downing MG, Biernacki K, et al (2019) Cognitive reserve and age predict cognitive recovery after mild to severe traumatic brain injury. J Neurotrauma 36:2753-2761. https://doi.org/10.1089/neu.2019.6430

27. Signorini DF, Andrews PJD, Jones PA, et al (1999) Predicting survival using simple clinical variables: a case study in traumatic brain injury. J Neurol Neurosurg Psychiatry 66:20-25. https://doi.org/10.1136/jnnp.66.1.20

28. Haveman ME, Van Putten MJAM, Hom HW, et al (2019) Predicting outcome in patients with moderate to severe traumatic brain injury using electroencephalography. Crit Care Lond Engl 23:401. https://doi.org/10.1186/s13054-019-2656-6

29. Lazaridis C, Yang M, DeSantis SM, et al (2015) Predictors of intensive care unit length of stay and intracranial pressure in severe traumatic brain injury. J Crit Care 30:1258-1262. https://doi.org/10.1016/j.jcrc.2015.08.003

30. Jensen JH, Helpern JA (2010) MRI quantification of non-Gaussian water diffusion by kurtosis analysis. NMR Biomed 23:698. https://doi.org/10.1002/nbm.1518

31. Zhang H, Schneider T, Wheeler-Kingshott CA, Alexander DC (2012) NODDI: practical in vivo neurite orientation dispersion and density imaging of the human brain. NeuroImage 61:10001016. https://doi.org/10.1016/j.neuroimage.2012.03.072

32. Provencio JJ, Hemphill JC, Claassen J, et al (2020) The Curing Coma Campaign: Framing Initial Scientific Challenges - Proceedings of the First Curing Coma Campaign Scientific Advisory Council Meeting. Neurocrit Care 33:1. https://doi.org/10.1007/s12028-020-01028-9

33. Izzy S, Compton R, Carandang R, et al (2013) Self-fulfilling prophecies through withdrawal of care: do they exist in traumatic brain injury, too? Neurocrit Care 19:347-363. https://doi.org/10.1007/s12028-013-9925-Z 
34. de Souza EM, Costa ET, Castellano G (2017) Phantoms for diffusion-weighted imaging and diffusion tensor imaging quality control: a review and new perspectives. Res Biomed Eng 33:156165. https://doi.org/10.1590/2446-4740.07816

35. Hicks R, Giacino J, Harrison-Felix C, et al (2013) Progress in developing Common Data Elements for traumatic brain injury research: Version Two - The end of the beginning. J Neurotrauma 30:1852-1861. https://doi.org/10.1089/neu.2013.2938 
Table 1. Characteristics of patients in the training dataset.

\begin{tabular}{|c|c|c|c|c|c|c|}
\hline & \multirow[b]{2}{*}{ All } & \multirow[b]{2}{*}{ FO } & \multirow[b]{2}{*}{ UFO } & \multicolumn{3}{|c|}{ Univariate logistic regression } \\
\hline & & & & Unit & Odds ratio $[95 \% \mathrm{CI}]$ & $\mathrm{P}$ value \\
\hline $\mathrm{N}$ & 50 & 20 & 30 & & & \\
\hline $\operatorname{Sex}(M / F)$ & $39 / 11$ & $15 / 5$ & $24 / 6$ & & & \\
\hline Median GOSE (IQR) & $3(4)$ & $5(1)$ & $1(2)$ & & & \\
\hline Mean age $(\mathrm{SD})^{\mathrm{a}}$ & $39.25(15.60)$ & $33.30(13.02)$ & $43.22(16.10)$ & per unit increase & $1.05[1.00-1.09]$ & $0.03 *$ \\
\hline Mean MRI delay $(\mathrm{SD})^{\mathrm{b}}$ & $21(8)$ & $23(7)$ & $20(8)$ & per unit decrease & $1.07[0.99-1.16]$ & 0.11 \\
\hline Mean LOS in ICU $(S D)^{c}$ & $53(25)$ & $53(17)$ & $54(29)$ & per unit increase & $1.00[0.98-1.03]$ & 0.84 \\
\hline Median GCS (IQR) & $4(4)$ & $5(5)$ & $4(3)$ & per unit decrease & $1.05[0.87-1.28]$ & 0.64 \\
\hline Mean $\overline{\mathrm{FA}}_{\text {deep }}(\mathrm{SD})$ & $0.85(0.08)$ & $0.90(0.04)$ & $0.82(0.09)$ & per 0.01 unit decrease & $1.33[1.12-1.58]$ & $0.001 *$ \\
\hline Mean $\overline{\mathrm{MD}}_{\text {deep }}(\mathrm{SD})$ & $1.04(0.07)$ & $1.04(0.04)$ & $1.04(0.09)$ & per 0.01 unit increase & $1.00[0.92-1.08]$ & 0.96 \\
\hline
\end{tabular}

UFO: unfavorable outcome; FO: favorable outcome; CI: confidence interval; N: number; M: male; F: female; GOSE: Glasgow outcome scale extended; IQR: interquartile range; SD: standard deviation; LOS: length of stay; ICU: intensive care unit; GCS: Glasgow Coma scale.

${ }^{a}$ Age is at the time of injury. Ages are expressed in years.

${ }^{\mathrm{b}}$ Delays are expressed in days after the injury.

${ }^{\mathrm{c}}$ Lengths of stay in ICU are expressed in days. Data were unavailable for 3 patients. 
Table 2. Characteristics of patients in the validation dataset.

\begin{tabular}{|c|c|c|c|c|c|c|c|}
\hline & All & & & MRI-COMA- & & CENTER-TB & \\
\hline & All & FO & UFO & FO & UFO & FO & UFO \\
\hline $\mathrm{N}$ & 196 & 94 & 102 & 46 & 94 & 48 & 8 \\
\hline $\operatorname{Sex}(M / F)$ & $155 / 41$ & $77 / 17$ & $78 / 24$ & $42 / 4$ & $74 / 20$ & $35 / 13$ & $4 / 4$ \\
\hline Median GOSE (IQR) & $3(5)$ & $6(2)$ & $1(2)$ & $5(2)$ & $1(2)$ & $6(2)$ & $3(1)$ \\
\hline Mean age $(\mathrm{SD})^{\mathrm{a}}$ & $\begin{array}{l}42.42 \\
(15.59)\end{array}$ & 39.83 (14.99) & $44.81(15.82)$ & $38.70(15.43)$ & $43.98(15.84)$ & $40.92(14.65)$ & $54.50(12.60)$ \\
\hline Mean MRI delay (SD) ${ }^{\mathrm{b}}$ & $19(7)$ & $18(7)$ & $19(6)$ & $19(8)$ & $20(6)$ & $18(6)$ & $14(8)$ \\
\hline $\begin{array}{l}\text { Mean LOS in ICU } \\
(\mathrm{SD})^{\mathrm{c}}\end{array}$ & $35(30)$ & $27(24)$ & $44(35)$ & $46(20)$ & $46(35)$ & $11(13)$ & $21(8)$ \\
\hline Median GCS (IQR) & $5(7)$ & $7(9)$ & $4(3)$ & $6(4)$ & $4(3)$ & $12(9)$ & $3(7)$ \\
\hline Mean $\overline{\mathrm{FA}}_{\text {deep }}(\mathrm{SD})$ & $0.87(0.09)$ & $0.92(0.06)$ & $0.81(0.08)$ & $0.89(0.05)$ & $0.80(0.08)$ & $0.96(0.06)$ & $0.90(0.08)$ \\
\hline Mean $\overline{\mathrm{MD}}_{\text {deep }}(\mathrm{SD})$ & $1.05(0.06)$ & $1.04(0.04)$ & $1.05(0.08)$ & $1.05(0.04)$ & $1.06(0.07)$ & $1.03(0.04)$ & $0.99(0.14)$ \\
\hline
\end{tabular}

UFO: unfavorable outcome; FO: favorable outcome; N: number; M: male; F: female; GOSE: Glasgow outcome scale extended; IQR: interquartile range; SD: standard deviation; LOS: length of stay; ICU: intensive care unit; GCS: Glasgow Coma scale.

${ }^{a} \mathrm{Age}$ is at the time of injury. Ages are expressed in years.

${ }^{\mathrm{b}}$ Delays are expressed in days after the injury.

${ }^{\mathrm{c}}$ Lengths of stay in ICU are expressed in days. Data were unavailable for 32 patients. 


\section{Figures}

Fig. 1 Flowchart showing how patients were included in the training and validation datasets and followed up

MRI-COMA

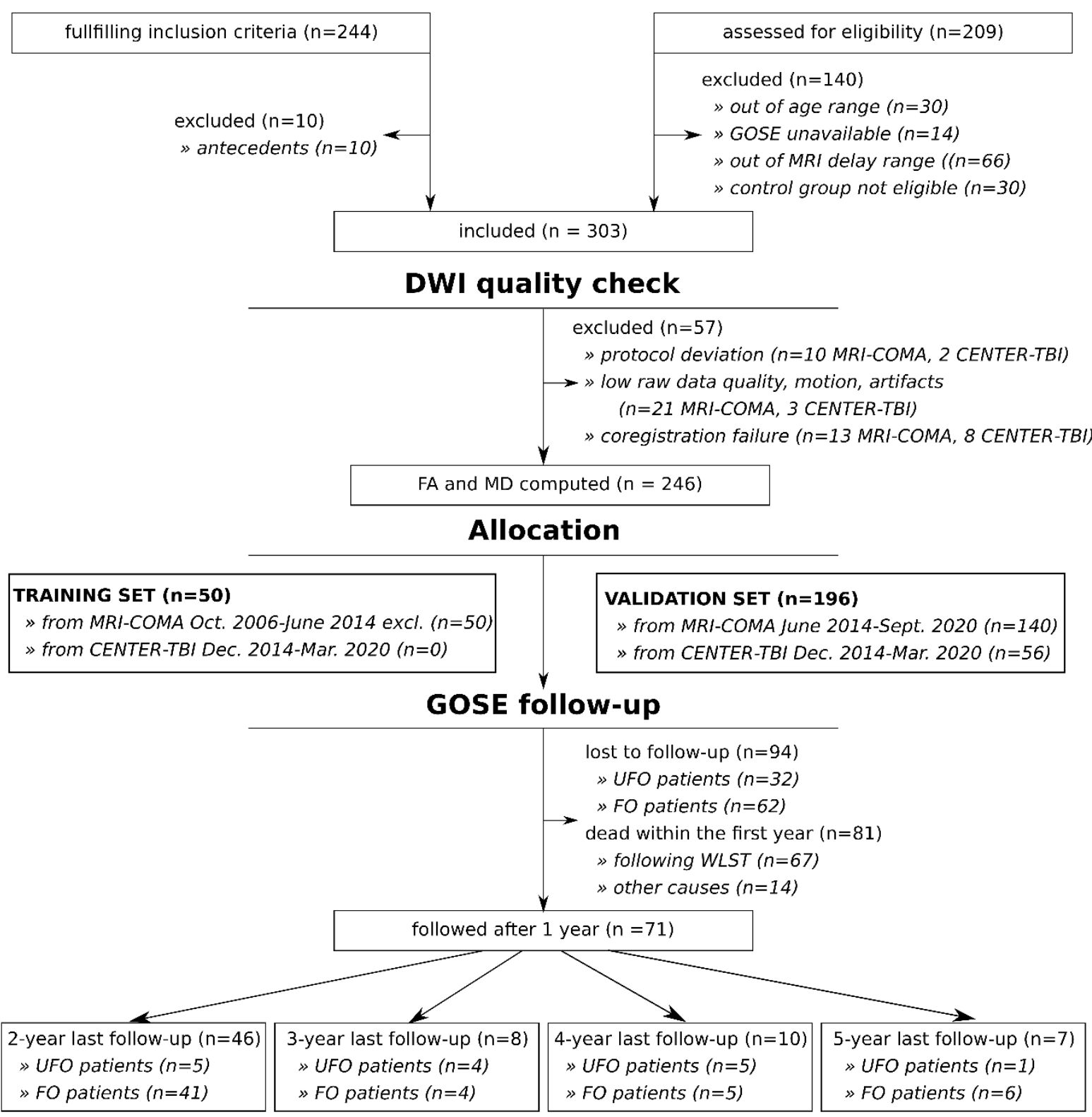


Fig. 2 Patients as a function of the prediction score CSCORE. Bars show the proportion of actual UFO patients in the corresponding CSCORE range. On the top of the figure, red dots (respectively, green ' + ') represent true UFO (respectively, FO) patients according to GOSE assessment one year after the injury. Cut-off value 0.19 delineates the left-hand-side red area where the specificity in predicting UFO is at least $99 \%$. Cut-off value 0.65 delineates the right-hand-side green area where the specificity in predicting FO is at least $95 \%$. Bottom: mean delay to MRI session in days ( \pm standard deviation) and median GCS (interquartile range) for patients in each area

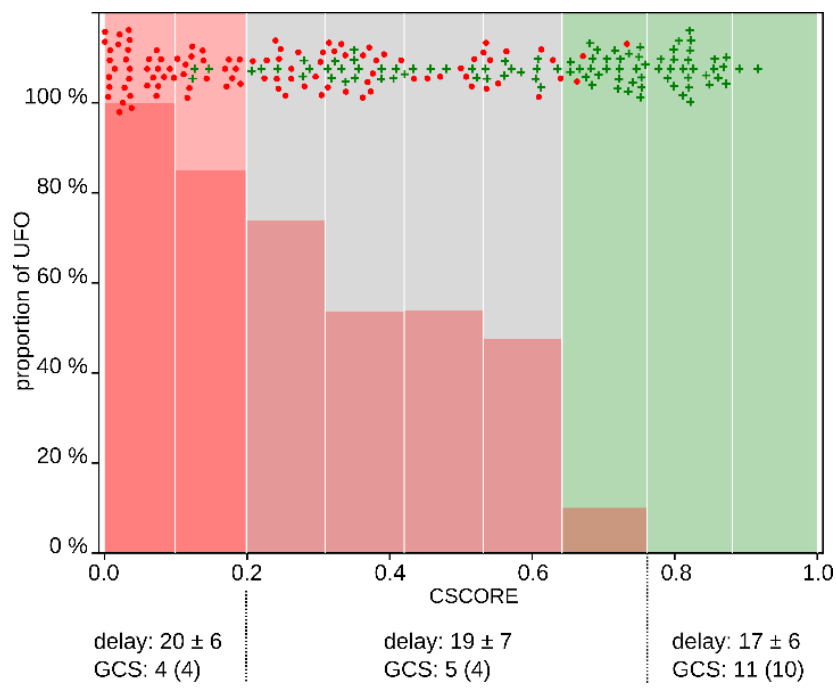


Fig. 3 Follow-up GOSE assessment from 1 to more than 5 years post-injury, for patients from both the training and the validation dataset for whom GOSE scores were available after 1 year, (a) predicted FO by the model (number of patients $\mathrm{N}=35)$, (b) in the "gray zone", predicted neither UFO nor FO (N=59), (c) predicted UFO $(\mathrm{N}=56)$. Each ribbon represents a patient. Hatched ribbons represent patients who underwent WLST

a.

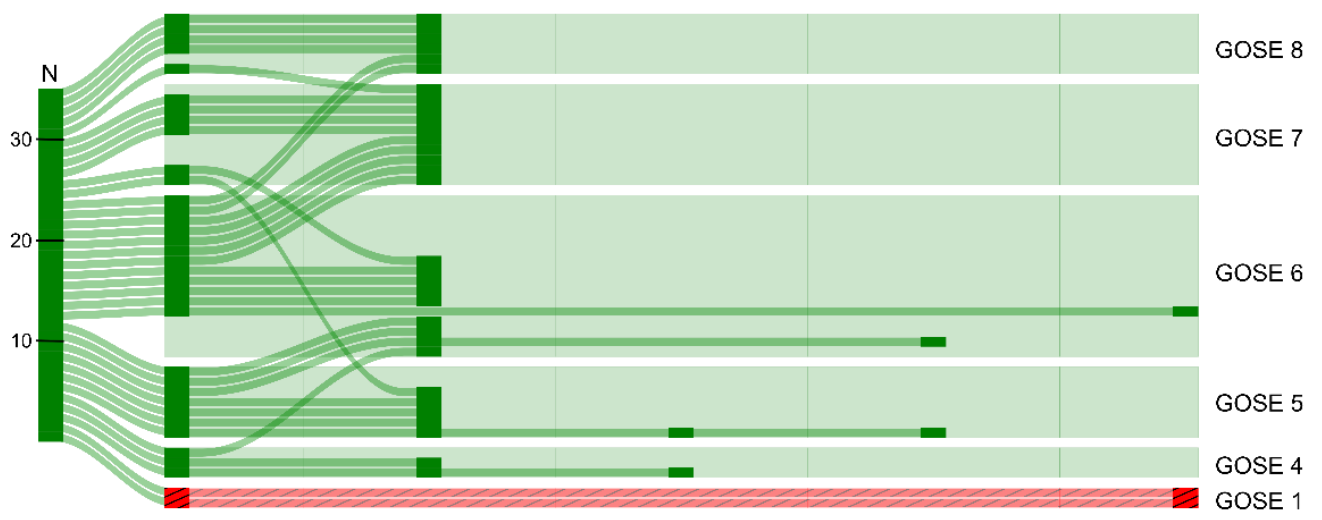

b.
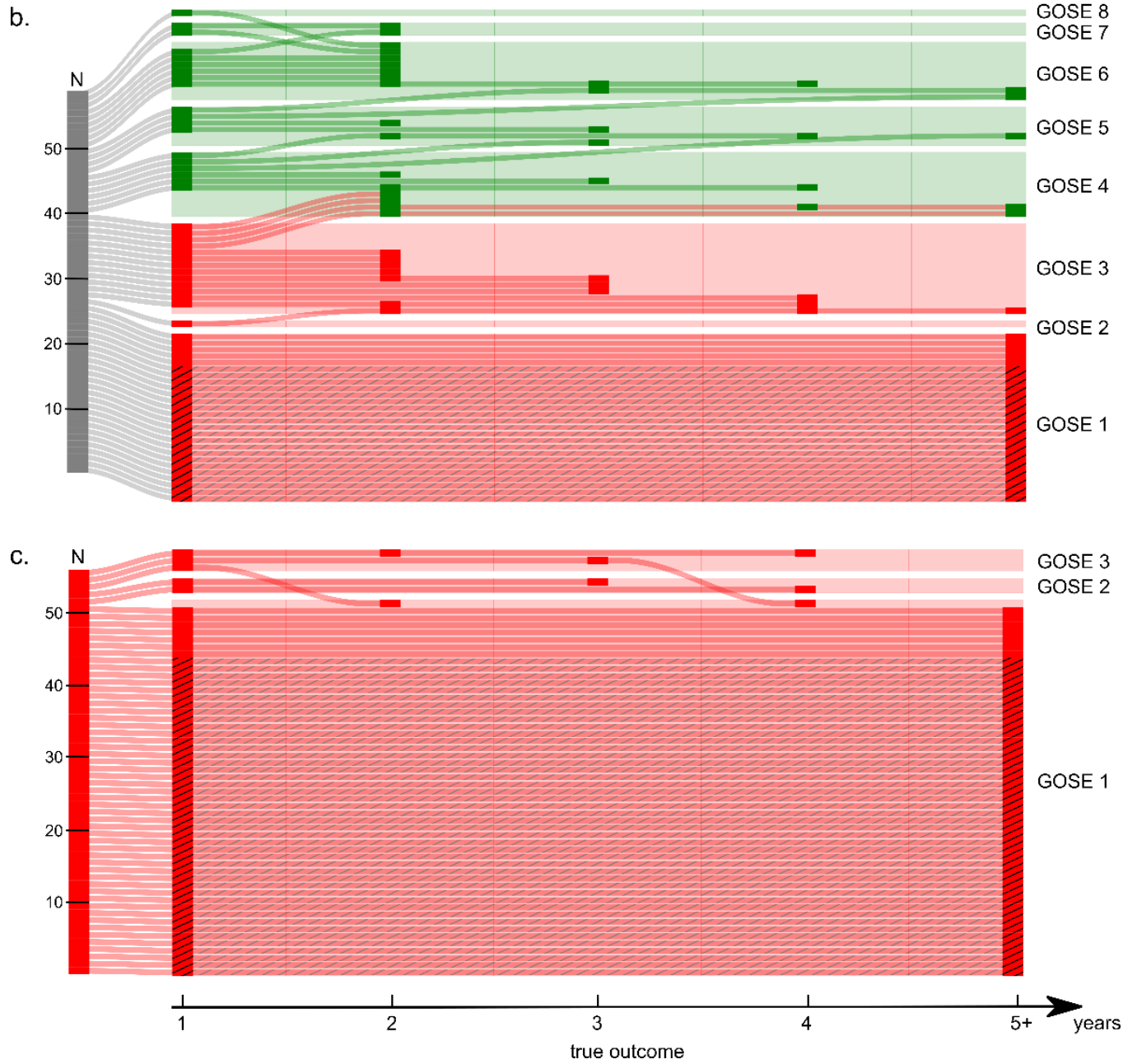